\title{
Neck Muscle
}

National Cancer Institute

\section{Source}

National Cancer Institute. Neck Muscle. NCI Thesaurus. Code C33163.

Any skeletal muscle located in the neck. 